RUB-TPII-18/05

\title{
Tagging the pion quark structure in QCD
}

\author{
A. P. Bakulev S. V. Mikhailov \\ Bogoliubov Laboratory of Theoretical Physics, \\ JINR, 141980 Dubna, Russia
}

(Dated: February 9, 2006)

\begin{abstract}
We combine the constraints on the pion quark structure available from perturbative QCD, nonperturbative QCD (nonlocal QCD sum rules and light cone sum rules) with the analysis of current data on $F_{\pi \gamma \gamma^{*}}\left(Q^{2}\right)$, including recent high-precision lattice calculations of the second moment of the pion's distribution amplitude. We supplement these constraints with those extracted from the renormalon approach by means of the twist-four contributions to the pion distribution amplitude in order to further increase stability with respect to related theoretical uncertainties. We show which regions in the space of the first two non-trivial Gegenbauer coefficients $a_{2}$ and $a_{4}$ of all these constraints overlap, tagging this way the pion structure to the highest degree possible at present.
\end{abstract}

PACS numbers: 12.38.Aw,12.38.Cy,12.38.Lg,13.40.Gp

The pion distribution amplitude (DA), $\varphi_{\pi}$, [1] plays a key role in hard-scattering QCD processes because it encapsulates the essential nonperturbative features of the pion's internal structure in terms of the partons' longitudinal momentum fractions $x_{i}$. Many studies $[2,[3,4,[5,6,6,8,9]$ have been performed in the literature to determine this DA using the high-precision CLEO data [10] on the pion-photon transition, or to infer it from the calculation of the corresponding form factor $F_{\pi \gamma^{*} \gamma}\left(Q^{2}\right)$. In particular, in [8] we have used light cone sum rules (LCSR) 3, 11] with a spectral density obtained in the standard factorization scheme at the nextto-leading-order (NLO) of perturbative QCD. We examined the theoretical uncertainties involved in the CLEOdata analysis in order to extract more reliably the first two non-trivial Gegenbauer coefficients $a_{2}$ and $a_{4}$, which parameterize the deviation from the asymptotic expression $\varphi_{\pi}^{\text {asy }}=6 x \bar{x}$ (where $\bar{x} \equiv 1-x$ ). A major outcome of this study [8] was that the best agreement with the data is provided by a pion DA with $a_{2}^{\text {best fit }}\left(\mu_{\mathrm{SY}}^{2}\right)=+0.23$ and $a_{4}^{\text {best fit }}\left(\mu_{\mathrm{SY}}^{2}\right)=-0.22$ at the scale $\mu_{\mathrm{SY}}^{2}=5.76 \mathrm{GeV}^{2}$ (Schmedding and Yakovlev [3]), where the twist-four contribution was represented by its asymptotic form. This best-fit solution (+) turns out to be very close to the model we derived before in [12] - dubbed BMS model (x) - from QCD sum rules with nonlocal condensates (NLC QCD SR) - see Fig. 11 Note that this model DA yields a prediction for $F_{\pi \gamma^{*} \gamma}\left(Q^{2}\right)$ that is in good agreement 13, 14 not only with the CLEO data, but also with the older low- $Q^{2}$ CELLO [15] data. The profile of this pion DA is double-humped, but has its endpoints $x=0$ and $x=1$ strongly suppressed. This suppression is directly related to the vacuum quark virtuality $\lambda_{q}^{2}$, whose

\footnotetext{
${ }^{\ddagger}$ On leave of absence from Institut für Theoretische Physik II, Ruhr-Universität Bochum, D-44780 Bochum, Germany

*Electronic address: bakulev@theor.jinr.ru

$\dagger$ Electronic address: mikhs@theor.jinr.ru

$\S$ Electronic address: stefanis@tp2.ruhr-uni-bochum.de
}

value $0.4 \mathrm{GeV}^{2}$, extracted from the CLEO data in [7], coincides with that used in 12], the reason being that, in the latter, it guarantees best stability of the NLC QCD

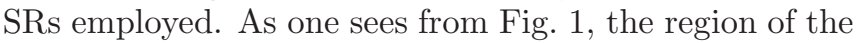
$a_{2}$ and $a_{4}$ values, admitted by the NLC QCD SRs (small slanted rectangle), is almost entirely enclosed by the $1 \sigma$ error ellipse. The exclusion of the Chernyak-Zhitnitsky (CZ) [16] pion DA ( $\square)$ and the asymptotic one $(\diamond)$, found before by Schmedding and Yakovlev [3] at the $2 \sigma$ level, was reinforced in [7]. In the updated analysis [8], which accounts for the variation of the size of the twist-four uncertainties more precisely, these pion DAs were found to be outside the $4 \sigma$ and $3 \sigma$ ellipses, respectively.

Recently, high-precision lattice measurements of the second moment $\left\langle\xi^{2}\right\rangle_{\pi}=\int_{0}^{1}(2 x-1)^{2} \varphi_{\pi}(x) d x$ of the pion DA were reported by two different collaborations [17, 18]. Both groups extracted from their respective simulations, values of $a_{2}$ at the scale $\mu_{\mathrm{SY}}^{2}$, but with different error bars. ${ }^{1}$ Unfortunately, the determination of the value of $a_{4}$ via the moment $\left\langle\xi^{4}\right\rangle_{\pi}$ appears to be a very difficult task on the lattice because it involves four covariant derivatives 17. It is remarkable that these lattice results are in striking agreement with the previous estimates of $a_{2}$ both from NLC QCD SRs [12] and also from the CLEO-data analyses - based on LCSR - 3, 7, 8], as illustrated in Fig. 1 where the lattice results are shown in the form of a vertical strip, containing the central value with associated errors. The (preliminary) results of the QCDSF/UKQCD collaboration [17] are shown in the left panels of Fig. 1 The right panels of this figure show the latest estimates by Del Debbio et al., reported in [18]. Previous determinations of the same team 19] favored the asymptotic DA, on the basis of much smaller values of the second moment $\left\langle\xi^{2}\right\rangle_{\pi}$. It seems that the

\footnotetext{
${ }^{1}$ We have evolved at the two-loop level both lattice results to the scale $\mu_{\mathrm{SY}}^{2}$ in order to facilitate comparison with the CLEO-data analyses.
} 

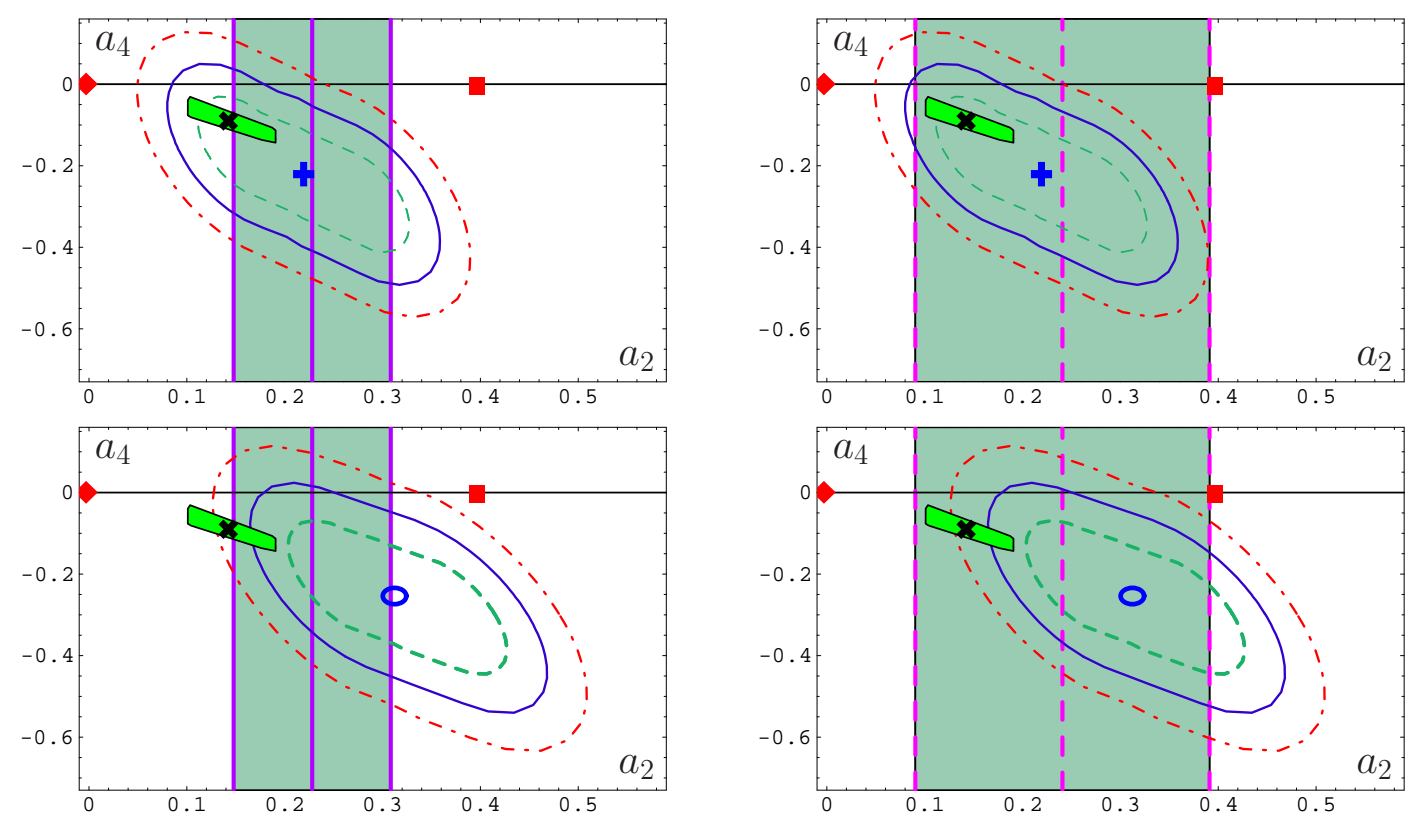

FIG. 1: The top figures show the LCSR-based CLEO-data analysis [7, 8] on $F_{\pi \gamma^{*} \gamma}\left(Q^{2}\right)$ in comparison with the lattice results of [17] (left panel) and 18] (right panel). The displayed models are explained in Table【 The bottom figures show the analogous results for the present data analysis which includes twist-four contributions by means of the renormalon approach. Here we use: dashed line: $1 \sigma$; solid line: $2 \sigma$; dash-dotted line: $3 \sigma$ ); slanted shaded rectangle: confidence region of NLC QCD SRs 12 for $\lambda_{q}^{2}=0.4 \mathrm{GeV}^{2}$. All results are evaluated at $\mu_{\mathrm{SY}}^{2}=5.76 \mathrm{GeV}^{2}$ after NLO ERBL evolution.

higher the precision of their simulation, the larger the result for $\left\langle\xi^{2}\right\rangle_{\pi}$ and, consequently, the more the asymptotic pion DA is disfavored. Remarkably, the value of $a_{2}$ of both displayed lattice measurements (middle line of each strip) is very close to the CLEO best fit in [7, 8] $(\mathbf{+})$, whereas the model pion DA (*) 12] is either inside the error margins (Del Debbio et al.), or just on the boundary (QCDSF/UKQCD collaboration).

But the success of the CLEO-data analysis depends crucially on the progress in reducing theoretical uncertainties originating from uncalculated (perturbative) higher-order radiative corrections and (mainly nonperturbative) higher-twist contributions. Those next-tonext-to-leading (NNLO) corrections proportional to the lowest $\beta$-function coefficient in the standard scheme were recently calculated in [20] and addressed in 8]. The higher-twist contributions encode information on powersuppressed corrections, related to higher quark-gluon Fock states and the quark transverse momentum. Just recently, Agaev [21] has attempted to improve the CLEOdata analysis by including twist-four contributions, specified by the renormalon approach [22, 23, 24]. The main aim was to estimate an upper bound for this contribution and extract new constraints on the Gegenbauer coefficients $a_{2}$ and $a_{4}$ of the leading twist-two pion DA. Agaev claims that the renormalon approach excludes negative values of $a_{4}$. This finding, remarkable if true, is incompatible with the results derived from the analyses in [3, 7, 8, 12], discussed above, and, therefore, deserves closer scrutiny.

Retracing Agaev's calculation, we found that it is seriously flawed in several respects. Firstly, we revealed that the normalization of the twist-four pion DAs is wrong because it erroneously depends on the Gegenbauer coefficient $a_{4}$ instead of being a constant. This might be the root cause for excluding negative values of $a_{4}$. Secondly, the Gegenbauer coefficients evolved to the scale $\mu_{\mathrm{SY}}^{2}$, given in his equation (4.2), turn out to be inconsistently low. These errors aside, this sort of approach looks quite attractive to be followed and rectified. In an effort to carry the renormalon approach to its logical conclusion and compare its predictions with the previous ones, we take recourse in the present investigation to the robust results of Braun-Gardi-Gottwald [24], which generalize the previous work of Andersen in 22], and embed their twist-four contributions to the pion DA into our data-processing framework [7, 8].

The starting point of our discussion is the twist expansion of the matrix element that defines the pion DAs (e.g., [25]):

$$
\begin{aligned}
\left\langle 0\left|\left[\bar{d}(z) \gamma_{\nu} \gamma_{5} \mathcal{W}(z, 0) u(0)\right]_{\mu_{\mathrm{F}}^{2}}\right| \pi^{+}(p)\right\rangle= & i f_{\pi} \int_{0}^{1} d x e^{-i \bar{x} p z}\left\{p_{\nu}\left[\varphi^{(2)}\left(x ; \mu_{\mathrm{F}}^{2}\right)+z^{2} \varphi_{1}^{(4)}\left(x ; \mu_{\mathrm{F}}^{2}\right)\right]\right. \\
& \left.+\left(z_{\nu}(p z)-p_{\nu} z^{2}\right) \varphi_{2}^{(4)}\left(x ; \mu_{\mathrm{F}}^{2}\right)\right\}+\mathcal{O}\left(z^{4}\right)
\end{aligned}
$$


where the scale $\mu_{\mathrm{F}}^{2}$ defines the factorization scale. Gauge invariance is ensured by the insertion of the light-like Wilson line $\mathcal{W}(z, 0)=P \exp \left[-i g \int_{0}^{1} d t z_{\mu} A^{\mu}(z-t z)\right]$. The twist-two contribution to the pion DA can be represented as an expansion in terms of Gegenbauer polynomials to read

$$
\varphi^{(2)}(x)=\sum_{n=0} a_{n} \psi_{n}(x) ; \psi_{n}(x)=6 x \bar{x} \cdot C_{n}^{3 / 2}(2 x-1) .
$$

For the leading twist-two part, we use a two-parameter model with the coefficients $a_{2}$ and $a_{4}$. This model receives support from nonlocal QCD sum rules 12] (cf. Fig. 11. At the reference scale $\mu_{\mathrm{SY}}^{2}$, we have after NLO evolution $a_{2}^{\mathrm{BMS}}=+0.14, a_{4}^{\mathrm{BMS}}=-0.09$. Note that though the NLO evolution generates higher Gegenbauer harmonics in the pion DA, the corresponding coefficients remain numerically negligible, so that the analysis can be based only on $a_{2}$ and $a_{4}$ 7]. To estimate the twist-four contribution, we now use the renormalon approach of Ref. 24]. To this end, we express the corresponding DA, $\Phi^{(4)}(x)$, characteristic for the reaction $\gamma^{*} \gamma \rightarrow \pi^{0}$ [1], with the aid of $\varphi^{(2)}(x)$, in the form of a convolution

$$
\begin{aligned}
\Phi^{(4)}\left(x ; \mu^{2}\right) & =\delta^{2}\left(\mu^{2}\right) K(x, y) \otimes \varphi^{(2)}(y) \\
& \equiv \delta^{2}\left(\mu^{2}\right) \int_{0}^{1} K(x, y) \varphi^{(2)}(y) d y,
\end{aligned}
$$

where the kernel $K$ is determined in the present work to be

$$
\begin{gathered}
K(x, y)=-\frac{2}{3}\left\{\theta(y>x)\left[\frac{x \bar{x}}{y^{2}}+\frac{1}{y} \ln \left(1-\frac{x}{y}\right)\right]\right. \\
+(x \rightarrow \bar{x}, y \rightarrow \bar{y})\} .
\end{gathered}
$$

In Eq. 3] the coupling $\delta^{2}\left(\mu^{2}\right)$ is defined by (see, for instance, [11]) $\left\langle\pi(p)\left|g_{\mathrm{s}} \bar{d} \tilde{G}_{\alpha \mu} \gamma^{\alpha} u\right| 0\right\rangle=i \delta^{2} f_{\pi} p_{\mu}$, where $\tilde{G}_{\alpha \mu}=(1 / 2) \varepsilon_{\alpha \mu \rho \sigma} G^{\rho \sigma}$ and $G_{\rho \sigma}=G_{\rho \sigma}^{a} \lambda^{a} / 2$. The normalization equation for the twist-four contribution, following from Eqs. (3) and (4), is

$$
\int_{0}^{1} d x K(x, y) \otimes \psi_{n}(y)=\frac{8}{9} \delta_{0 n}
$$

It is important to notice in this context that Eq. (3) accumulates the content of the twist-four pion DAs, termed $\varphi_{1}^{(4)}, \quad \varphi_{2}^{(4)}, \Phi_{\|}, \quad \Psi_{\|}$in [21] (Eq. (3.4) there)-see also Eq. (20) in 11]. Explicit expressions for these DAs can be found in 24] (Eqs. (3.4) and (3.5)). Here we display the explicit expression for the twist-four DA we have derived:

$$
\begin{aligned}
\Phi^{(4)}\left(x ; \mu^{2}\right)=-4 \delta^{2}\left(\mu^{2}\right)\{ & x^{2} \ln (x)\left(1+a_{2}+a_{4}\right)-5 a_{2} x \bar{x}\left[\frac{1}{2}-\frac{1}{4} x \bar{x}+2 x \ln (x)\right] \\
& \left.-7 a_{4} x \bar{x}\left[1+\frac{35}{8} x \bar{x}-\frac{47}{4}(x \bar{x})^{2}+\left(13-27 x+18 x^{2}\right) x \ln (x)\right]+(x \rightarrow \bar{x})\right\} .
\end{aligned}
$$

We estimate within this renormalon-based approach error ellipses of the CLEO data and show the results of the data processing in Figs. 1 and 2 As one sees from these figures, the general effect, entailed by the inclusion of the twist-four contributions by means of the renormalon approach, is to shift the error ellipses further to the right, somewhat enlarging them as well. One observes that larger values of $a_{2}$ and $a_{4}$ are preferred, while, at the considered $1 \sigma$ error level, the sign of $a_{4}$ remains unchanged and negative. This phenomenon is in agreement with what we had found before in [8], see Table 1 there, when we varied the twist-four coupling parameter $\delta\left(\mu^{2}\right)$ from smaller to larger values.

For the new best-fit point $(\mathrm{O})$, we obtain the following values $a_{2}^{\text {best fit(ren) }}\left(\mu_{\mathrm{SY}}^{2}\right)=+0.31$ and $a_{4}^{\text {best fit(ren) }}\left(\mu_{\mathrm{SY}}^{2}\right)=$ -0.25 . Note that the $\chi^{2}$ value of that best-fit point $\left(\chi_{\text {best fit(ren })}^{2}=0.58\right)$ is comparable with the one in the standard analysis $\left[7,[8]\left(\chi_{\text {best fit }}^{2}=0.47\right)\right.$. Analyzing the structure of the twist-four pion DA, given by Eq. (6), one finds that it has two distinctive peaks and strongly enhanced endpoint regions $x=0,1$, in accordance to the assertions in 24] and 21]. But, perhaps somewhat surprisingly, including this contribution into the LCSR analysis of the CLEO data, the characteristics of the best-fit pion DA in the endpoint region remain almost the same as found before [7, 8] with the use of the asymptotic form for $\Phi^{(4)}\left(x ; \mu^{2}\right)$. Both DAs show endpoint suppression, much like the BMS model DA extracted from the NLC QCD SRs 12], though these two approaches are not related to each other. Given that the renormalon-based twist-four contribution represents an upper bound 24], we can conclude that the endpoint region of the pion DA will not receive further enhancement.

Figure 2 presents a collage of several proposed models for the pion DA, listed in Table凹 in comparison with the CLEO-data constraints in terms of the $1 \sigma$ ellipses, and the recent lattice measurements of Refs. 17, 18]. The influence of NNLO radiative corrections - only partially known [20] - can be investigated by means of varying the 
TABLE I: Estimates at the normalization scale $\mu_{0}^{2}=1 \mathrm{GeV}^{2}$ for the Gegenbauer coefficients and the reduced inverse moment $\left\langle x^{-1}\right\rangle_{\pi}^{\mathrm{R}}$ for several model DAs for the pion, based on the first two Gegenbauer coefficients $a_{2}$ and $a_{4}$. The designations correspond to those used in Fig. 2 Included are also the theoretical constraints derived from LCSRs and NLC QCD SRs, and by analyzing [7] the CLEO data [10]. Also shown are the lattice measurements of [18] and [17] and the previous transverselattice result. [Note that the uncertainties on the Gegenbauer coefficients $a_{2}$ and $a_{4}$ are correlated. Here, the rectangular limits of the fiducial ellipse [7, 8] are shown.]

\begin{tabular}{|c|c|c|c|c|}
\hline DA models/methods & Symbols & $a_{2}\left(\mu_{0}^{2}\right)$ & $a_{4}\left(\mu_{0}^{2}\right)$ & $\left\langle x^{-1}\right\rangle_{\pi}^{\mathrm{R}}$ \\
\hline As & $\diamond$ & 0 & 0 & 0 \\
\hline $\mathrm{ADT}[26]$ & $\triangle$ & 0.05 & -0.04 & 0.01 \\
\hline BMS best fit point [7] & + & 0.31 & -0.35 & -0.04 \\
\hline Best fit point (ren) & O & 0.44 & -0.40 & 0.04 \\
\hline PPRWG [27] & 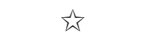 & 0.046 & 0.007 & 0.05 \\
\hline $\mathrm{SY}[25]$ & $\bullet$ & 0.27 & -0.22 & 0.05 \\
\hline BMS [12] & $\boldsymbol{x}$ & 0.20 & -0.14 & 0.06 \\
\hline $\mathrm{PR}[28]$ & $\nabla$ & 0.09 & -0.02 & 0.07 \\
\hline $\mathrm{BZ}$ [29] & $\boldsymbol{\Delta}$ & 0.12 & -0.02 & 0.10 \\
\hline Agaev [9] & $\diamond$ & 0.23 & -0.05 & 0.18 \\
\hline $\mathrm{CZ}[16]$ & $\mathbf{\square}$ & 0.56 & 0 & 0.56 \\
\hline $\mathrm{BF}[25]$ & $\square$ & 0.44 & 0.25 & 0.69 \\
\hline NLC QCD SRs for $\left\langle\xi^{N}\right\rangle_{\pi}[8,12]$ & 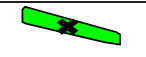 & {$[0.13,0.25]$} & {$[-0.04,-0.22]$} & - \\
\hline "Daughter" NLC QCD SR [8, 12] & & - & - & $0.09 \pm 0.10$ \\
\hline LCSR analysis of JLab data [30] & & $0.24 \pm 0.14 \pm 0.08$ & - & - \\
\hline LCSR analysis of CLEO data $[8,10]$ & & - & - & $-0.03 \pm 0.18$ \\
\hline Transverse lattice [31] & $\boldsymbol{\nabla}$ & 0.08 & 0.02 & 0.10 \\
\hline QCDSF/UKQCD lattice [17] & 1 & $0.32 \pm 0.11$ & - & $" 0.32 \pm 0.11 "$ \\
\hline Del Debbio et al. (lattice) [18] & 1 & $0.34 \pm 0.21$ & - & $" 0.34 \pm 0.21 "$ \\
\hline
\end{tabular}

factorization scale, say, in the interval $Q^{2} / 2 \leq \mu^{2} \leq 2 Q^{2}$, where $\mu^{2}=\mu_{\mathrm{F}}^{2}=\mu_{\mathrm{R}}^{2}$. This has been discussed in [8] and it has been shown that it does not alter qualitatively the main results. Note that the NNLO corrections within the conformal scheme have been quantitatively analyzed in [20]. Looking more carefully on Fig. 2] one recognizes an interesting pattern: with the exception of the $\mathrm{BF}$ model and the $\mathrm{CZ}$ one, all other models steer themselves along an "orbit" defined by $a_{2}+a_{4} \approx$ const. The organizing tool behind that pattern is the reduced inverse moment $\left\langle x^{-1}\right\rangle_{\pi}^{\mathrm{R}}$ of the pion DA,

$$
\left\langle x^{-1}\right\rangle_{\pi}^{\mathrm{R}} \equiv \frac{\left\langle x^{-1}\right\rangle_{\pi}}{3}-1=a_{2}+a_{4}+\ldots,
$$

where the ellipsis stands for still higher Gegenbauer coefficients. This inverse moment plays a special role in the description of several form factors of the pion in perturbative QCD.

The rationale for it is as follows. Though the diversity in shape among the models shown in the first column of Table [-in correspondence with Fig. 2-is substantial, the associated values for the reduced inverse moment are relatively close to each other (see the last column of Table 【). Nevertheless, the predictions for the pion-photon transition form factor vary quite strongly along this orbit as it is evident from Fig. 2] The deep reason for this, is that this form factor is not directly proportional to the reduced inverse moment (for more details, the interested reader is referred to Appendix E of [7] and to [12]).

In addition, as it can be shown by using the techniques developed in 32, 33], if the reduced inverse moment is in the range of the NLC QCD SRs (see Table I), then also the hard contribution to the electromagnetic pion form factor has the right magnitude - provided one uses for all model DAs, the same soft contribution, say, that employed in [32, 33] on account of quark-hadron local duality. Moreover, the estimate $\left\langle x^{-1}\right\rangle_{\pi}^{\mathrm{EM}} / 3-1=0.24 \pm$ 0.16 , obtained in the data analysis of the electromagnetic (EM) pion form factor within the framework of a different LCSR method in 30,34 - though only dependent on $a_{2}$-is compatible within errors with the quoted NLC QCD SR result. On that basis, one might expect that future lattice measurements should yield negative values of $a_{4}$ in order to be in line with the typical value of the reduced moment. This would decrease the values shown in Table凹in quotation marks, recalling that they contain only the first Gegenbauer coefficient $a_{2}$.

We have given a comprehensive compendium of the current situation on the constraints imposed on the first two non-trivial Gegenbauer coefficients by the analysis of the CLEO data 10] in comparison with the most recent estimates from modern lattice technology [17, 18]. We 
used in our data analysis the method of light-cone sum rules including, on one hand, perturbative corrections up to NLO, and, on the other hand, taking into account the twist-four contribution to the pion DA, having recourse to the renormalon approach. We believe that this investigation further restricts the available space of values in the $\left(a_{2}, a_{4}\right)$ plane for the pion DA, confirming the negative sign of the Gegenbauer coefficient $a_{4}$ at the $1 \sigma$ level of the CLEO-data analysis without and with the inclusion of the twist-four contribution via the renormalon method. The gross profile emerging from this CLEOdata analysis is consistent with that we have determined independently some years ago [12] from QCD sum rules with nonlocal condensates. In fact, all constraints we have presented above are consistent with a profile for the pion DA that has two humps, but with strongly suppressed endpoints at $x=0$ and $x=1$. The endpointenhancement of the renormalon-based twist-four contribution does not change these characteristics. We emphasize that this type of pion DA leads to a pion-photon transition form factor that was found [13, 14 to be in good agreement with the (low) $Q^{2}$ CELLO data 15] as well. A full-blown reconstruction of the pion DA in terms of its Gegenbauer decomposition may become possible in the next years, once the lattice community can provide estimates for $a_{4}$ that seems quite difficult at present. Further advances in the calculation of the higher-twist contributions to the pion DA and inclusion of the partial NNLO radiative corrections in the standard scheme 20] will also improve the theoretical precision in assessing the pion structure via the CLEO or other data of similar precision. In this respect, a combined use of data on the pion-photon transition with such on the electromagnetic pion form factor, after the upgrade to $12 \mathrm{GeV}$, of the CEBAF machine at JLab, will considerably help to tag the pion structure at a still deeper level.

Two of us (A.P.B. and S.V.M.) are indebted to Prof. Klaus Goeke for the warm hospitality at Bochum University, where part of this work was carried out. N.G.S.

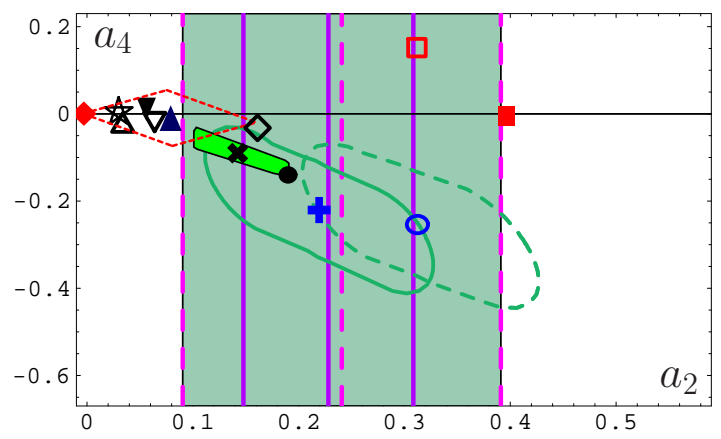

FIG. 2: Collage of several proposed models, compiled in Table $\square$ in comparison with the CLEO data and lattice measurements. The dashed rhombus containing the BZ point represents the BZ constraints [29]. All results are evaluated at $\mu_{\mathrm{SY}}^{2}=5.76 \mathrm{GeV}^{2}$ after NLO ERBL evolution.

thanks for support the BLTP@JINR, where this work was completed. This investigation was supported in part by the Heisenberg-Landau Programme (grant 2005) and the Russian Foundation for Fundamental Research (grant No. 03-02-04022).
[1] A. V. Radyushkin, Dubna preprint P2-10717, 1977 hep-ph/0410276.

[2] P. Kroll and M. Raulfs, Phys. Lett. B387, 848 (1996).

[3] A. Schmedding and O. Yakovlev, Phys. Rev. D62, 116002 (2000).

[4] N. G. Stefanis, W. Schroers, and H.-C. Kim, Phys. Lett. B449, 299 (1999); Eur. Phys. J. C18, 137 (2000).

[5] E. R. Arriola and W. Broniowski, Phys. Rev. D66, 094016 (2002).

[6] A. P. Bakulev and S. V. Mikhailov, Phys. Rev. D65, 114511 (2002).

[7] A. P. Bakulev, S. V. Mikhailov, and N. G. Stefanis, Phys. Rev. D67, 074012 (2003).

[8] A. P. Bakulev, S. V. Mikhailov, and N. G. Stefanis, Phys. Lett. B578, 91 (2004).

[9] S. S. Agaev, Phys. Rev. D 72, 114010 (2005).

[10] J. Gronberg et al., Phys. Rev. D57, 33 (1998).

[11] A. Khodjamirian, Eur. Phys. J. C6, 477 (1999).

[12] A. P. Bakulev, S. V. Mikhailov, and N. G. Stefanis, Phys. Lett. B508, 279 (2001); [Erratum-ibid. B 590, 309 (2004)]; in Proceedings of the 36th Rencontres De Moriond on QCD and Hadronic Interactions, 17-24 Mar 2001, Les Arcs, France, edited by J. T. T. Van (World Scientific, Singapore, 2002), pp. 133-136.

[13] A. P. Bakulev, S. V. Mikhailov, and N. G. Stefanis, Phys.
Part. Nucl. 35, 7 (2004).

[14] A. P. Bakulev, S. V. Mikhailov, and N. G. Stefanis, Annalen Phys. 13, 629 (2004).

[15] H. J. Behrend et al., Z. Phys. C49, 401 (1991).

[16] V. L. Chernyak and A. R. Zhitnitsky, Phys. Rept. 112, 173 (1984).

[17] M. Göckeler et al., hep-lat/0510089

[18] L. Del Debbio, Few Body Syst. 36, 77 (2005).

[19] L. Del Debbio, M. Di Pierro, and A. Dougall, Nucl. Phys. Proc. Suppl. 119, 416 (2003).

[20] B. Melić, D. Müller, and K. Passek-Kumerički, Phys. Rev. D68, 014013 (2003).

[21] S. S. Agaev, hep-ph/0511192

[22] J. R. Andersen, Phys. Lett. B475, 141 (2000).

[23] S. S. Agaev, Phys. Rev. D69, 094010 (2004).

[24] V. M. Braun, E. Gardi, and S. Gottwald, Nucl. Phys. B685, 171 (2004).

[25] V. M. Braun and I. E. Filyanov, Z. Phys. C44, 157 (1989).

[26] I. V. Anikin, A. E. Dorokhov, and L. Tomio, Phys. Part. Nucl. 31, 509 (2000).

[27] V. Y. Petrov, M. V. Polyakov, R. Ruskov, C. Weiss, and K. Goeke Phys. Rev. D59, 114018 (1999).

[28] M. Praszalowicz and A. Rostworowski, Phys. Rev. D64, 074003 (2001); Phys. Rev. D66, 054002 (2002). 
[29] P. Ball and R. Zwicky, Phys. Lett. B625, 225 (2005); Phys. Rev. D71, 014015 (2005).

[30] J. Bijnens and A. Khodjamirian, Eur. Phys. J. C26, 67 (2002).

[31] S. Dalley and B. van de Sande, Phys. Rev. D67, 114507 (2003).

[32] A. P. Bakulev, K. Passek-Kumerički, W. Schroers, and N. G. Stefanis, Phys. Rev. D70, 033014 (2004).

[33] N. G. Stefanis et al., in First International Workshop
"Hadron Structure and QCD (HSQCD 2004): From Low to High Energies", Repino, St. Petersburg, Russia, 1822 May 2004, edited by V. T. Kim and L. N. Lipatov (PNPI, Gatchina, St. Petersburg, 2004), pp. 238-245 hep-ph/0409176.

[34] V. M. Braun, A. Khodjamirian, and M. Maul, Phys. Rev. D61, 073004 (2000). 